DOI: https://doi.org/10.34883/PI.2021.13.5.015

УДК 616.12-008.33

Казакова М.И., Галицкая С.С.

Республиканский клинический медицинский центр Управления делами Президента

Республики Беларусь, Минск, Беларусь

Kazakova M., Galitskaya S.

Republican Clinical Medical Center of the Administrative Department of the President

of the Republic of Belarus, Minsk, Belarus

\title{
Первичный гиперальдостеронизм
}

\section{как причина рефрактерной артериальной гипертензии. Клинический случай}

\author{
Primary Hyperaldosteronism as a Cause of Refractory Arterial \\ Hypertension. A Clinical Case
}

Резюме

В последние годы достигнут значительный прогресс в понимании патогенеза первичного гиперальдостеронизма (ПГА), расширены показания для проведения скрининга, разработаны алгоритмы для медикаментозного и хирургического лечения. У пациентов с ПГА более высокий риск развития инфаркта миокарда, кардиального фиброза, диастолической дисфункции, гипертрофии миокарда левого желудочка, дилатации полостей сердца и возникновения аритмий по сравнению с пациентами с эссенциальной артериальной гипертензией. Риск внезапной сердечной смертности у этих пациентов увеличивается в 10-12 раз. Своевременно поставленный диагноз ПГА позволяет предотвратить развитие осложнений, улучшить качество жизни и снизить инвалидизацию пациентов.

В статье представлен клинический случай диагностики и лечения первичного гиперальдостеронизма как наиболее частой причины вторичной артериальной гипертензии. Стертая клиническая картина в дебюте заболевания и отсутствие гипокалиемии маскируют первичный гиперальдостеронизм под эссенциальную гипертензию и приводят к позднему выявлению заболевания.

Ключевые слова: первичный гиперальдостеронизм, альдостерон, ренин, альдостерон-рениновое соотношение, гипокалиемия.

\section{Abstract}

In recent years, significant progress has been made in understanding the pathogenesis of primary hyperaldosteronism (PHA). The indications for screening have been expanded; the algorithms for drug and surgical treatment have been developed. Patients with PHA have a higher risk of development of myocardial infarction, cardiac fibrosis, diastolic dysfunction, left ventricular hypertrophy, dilation of the heart cavities, and occurrence of arrhythmias if compared with patients with essential arterial hypertension. The risk of sudden cardiac death in these patients increases by 10-12 times. A timely diagnosis of PHA can prevent the development of complications, improve the quality of life, and reduce the disability of patients. 
The article presents a clinical case of diagnosis and treatment of primary hyperaldosteronism as the most common cause of secondary arterial hypertension. A blurred clinical picture at the onset of the disease and the absence of hypokalemia mask primary hyperaldosteronism as essential hypertension and lead to late detection of disease.

Keywords: primary hyperaldosteronism, aldosterone, renin, aldosterone-renin ratio, hypokalemia.

\section{- ВВЕДЕНИЕ}

Первичный гиперальдостеронизм (ПГА) - клинический синдром, который возникает при избыточной секреции альдостерона функционально автономным от ренин-ангиотензин-альдостероновой системы опухолевым или гиперпластическим процессом в коре надпочечника [1].

Исторически ПГА рассматривался как редкая причина развития вторичной (эндокринной) артериальной гипертензии, а важными диагностическими критериями являлись гипокалиемия и наличие образования надпочечника. Однако последние исследования показывают, что ПГА является распространенным состоянием, которое часто остается недиагностированным [2].

Распространенность ПГА среди пациентов с артериальной гипертензией составляет 6\% [3], а у пациентов со стойкой артериальной гипертензией ПГА наблюдается в 12-20\% случаев [4].

В самом крупном на сегодняшний день исследовании только половина пациентов с альдостерон-продуцирующей аденомой (АПА) и $17 \%$ пациентов с идиопатическим гиперальдостеронизмом (ИГА) имели концентрацию калия в сыворотке крови $<3,5$ ммоль/л. Таким образом, именно нормокалиемическая гипертензия чаще встречается при ПГА, а гипокалиемия, вероятно, присутствует только в более тяжелых случаях [5].

Следовательно, наличие гипокалиемии имеет низкую чувствительность, а отсутствие гипокалиемии имеет низкую отрицательную прогностическую ценность для диагностики ПГА [6].

Клиническое значение ПГА связано с кардиометаболическими последствиями высокой продукции альдостерона. Пациенты с ПГА имеют более высокий риск развития ишемической болезни сердца, фибрилляции предсердий, инсульта, гипертрофии миокарда левого желудочка, сердечной недостаточности, метаболического синдрома, чем сопоставимые по возрасту и полу пациенты с эссенциальной гипертензией и той же степенью повышения артериального давления [7, 8].

Таким образом, крайне важно, чтобы пациенты с ПГА были выявлены на ранней стадии, что позволит вовремя начать специфическую терапию и избежать осложнений.

Показания к скринингу на ПГА (по данным клинических рекомендаций Международного эндокринологического общества 2016 г.):

- артериальное давление $>150 / 100$ мм рт. ст. при 3 последовательных измерениях в разные дни;

- артериальное давление $>140 / 90$ мм рт. ст., устойчивое к 3 антигипертензивным препаратам, включая диуретики; 
- артериальное давление $<140 / 90$ мм рт. ст. при приеме 4 или более антигипертензивных препаратов;

- артериальная гипертензия и гипокалиемия (в т. ч. индуцированная приемом диуретиков);

- артериальная гипертензия с инциденталомой надпочечника;

- артериальная гипертензия с синдромом обструктивного апноэ;

- артериальная гипертензия и отягощенный семейный анамнез по наличию гипертензии или инсульта в молодом возрасте (до 40 лет);

- артериальная гипертензия и наличие ПГА у родственников 1-й степени родства [9].

\section{Диагностика}

ПГА диагностируется на основании исследования альдостерона, активности ренина плазмы (или прямой концентрации ренина) и значения альдостерон-ренинового соотношения (АРС).

Определение АРC производится в утренние часы (после пребывания пациента в вертикальном положении не менее 2 часов), перед забором крови пациент должен сидеть в течение 5-15 минут. Перед выполнением теста пациент не должен придерживаться бессолевой диеты. Так как гипокалиемия уменьшает секрецию альдостерона, она должна быть скорректирована до определения АРС.

Антагонисты минералокортикоидных рецепторов (спиронолактон, эплеренон) желательно отменить за 4 недели, а другие препараты, влияющие на ренин-ангиотензиновую систему (РАС), - за 2 недели. Предпочтительно определять АРС на фоне приема недигидропиридиновых блокаторов кальциевых каналов (верапамил) или блокаторов а-адренорецепторов (доксазозин). Однако в клинической практике иногда невозможно отменить препараты, влияющие на РАС, из-за неконтролируемого артериального давления. В таких случаях допустимо проводить определение АРС на фоне приема этих препаратов. Результат необходимо интерпретировать с учетом принимаемых лекарств.

При сочетании спонтанной гипокалиемии, уровня альдостерона $>20$ нг/дл (550 пмоль/л) и неопределяемого уровня ренина (или его активности) ПГА предлагается считать установленным без проведения дальнейшей подтверждающей диагностики.

Компьютерная томография (КТ) рекомендована всем пациентам с ПГА, даже если не планируется или противопоказано оперативное лечение, чтобы исключить адренокортикальную карциному.

Сравнительный селективный венозный забор крови (ССВЗК) - это «золотой стандарт» диагностики для выявления одностороннего или двустороннего характера секреции альдостерона у пациентов с ПГА и уточнения показаний к оперативному лечению.

\section{Лечение}

Методом выбора в лечении подтипов ПГА с односторонней гиперпродукцией альдостерона (АПА и ОГН) является лапароскопическая адреналэктомия. При высоком риске операции или отказе пациента от операции рекомендовано лечение антагонистами минералокортикоидных рецепторов (спиронолактон, эплеренон). 


\section{- КЛИНИЧЕСКИЙ СЛУЧАЙ}

Пациентка М., 56 лет, поступила в кардиологическое отделение государственного учреждения «Республиканский клинический медицинский центр» Управления делами Президента Республики Беларусь с жалобами на выраженную общую слабость, утомляемость, перебои в работе сердца, сердцебиение и одышку при незначительных нагрузках, повышение артериального давления. Ухудшение самочувствия отмечает в течение 6 месяцев (появились перебои в работе сердца, приступы учащенного сердцебиения, появилась и усилилась со временем общая слабость). За месяц до госпитализации у пациентки появились одышка и плохая переносимость бытовых нагрузок из-за выраженной слабости.

В анамнезе у пациентки артериальная гипертензия (около 15 лет) с частыми гипертоническими кризами. Гипотензивные препараты принимает регулярно (амлодипин 10 мг/сутки, периндоприл 8 мг/сутки, индапамид 2,5 мг/сутки, моксонидин 0,2 мг/сутки), целевые уровни АД на этом фоне не достигнуты. В амбулаторной карте, предоставленной для анализа, минимальное артериальное давление, зарегистрированное в кабинете врача, - 155/95 мм рт. ст., а резкое повышение артериального давления наблюдается 3-4 раза в месяц (максимально до 240/130 мм рт. ст.). Со слов пациентки, показатели АД, измеренного дома, находятся в диапазоне 150/95-165/100 мм рт. ст.

За 4 месяца до госпитализации у пациентки было выявлено нарушение ритма по типу полиморфной желудочковой экстрасистолии, неустойчивых пароксизмов желудочковой тахикардии (по ЭКГ). В связи с этим была выполнена коронароангиография (коронарные артерии без стенозов). Для лечения аритмии пациентке был назначен амиодарон 200 мг 2 раза в сутки. По материнской линии наследственность отягощена по ишемической болезни сердца, артериальной гипертензии и инфаркту миокарда. Статус курения и привычного употребления алкоголя отрицательный.

Диагноз при направлении в стационар: постмиокардитический кардиосклероз. Пароксизмальная неустойчивая желудочковая тахикардия, частая полиморфная желудочковая экстрасистолия. Н1. Артериальная гипертензия 2-й степени, риск 4.

\section{Обследование}

Пациентка нормостенического телосложения. ИМТ 23,3 кг/м². Окружность талии 78 см. Костно-мышечная система без видимых изменений. АД, измеренное в положении сидя на двух руках, 180/80-190/80 мм рт. ст. Аускультативно тоны сердца аритмичные (частая экстрасистолия), ЧСС 50 уд/мин.

При поступлении в биохимическом анализе выраженные электролитные нарушения, повышение мочевины, креатинина: калий 2,37 ммоль/л (N 3,5-5,1), натрий 148 ммоль/л (N 135-145), хлориды 91,9 ммоль/л (N 98-107), креатинин 168 мкмоль/л (N 44-80), мочевина 9,1 ммоль/л (N 2,76-8,07), СКФ (CKD-EPI 34 мл/мин/1,73 м²). КЩС: pH 7,48 $(7,35-7,45)$.

NTproBNP 904 пг/мл (N 0-100).

ЭКГ при поступлении: синусовый ритм. Горизонтальное положение ЭОС. Частые по типу бигеминии одиночные желудочковые 
экстрасистолы. Неполная блокада правой ножки пучка Гиса. чСС 56 уд/мин.

Суточный мониторинг ЭКГ при поступлении проводился на фоне длительного амбулаторного приема амиодарона 400 мг/сутки (амиодарон отменен при поступлении, $\beta$-адреноблокаторы не принимала): синусовый ритм с эпизодами брадикардии в дневное и ночное время суток. Частые с эпизодами бигеминии одиночные желудочковые экстрасистолы (2017/сутки), парные политопные желудочковые экстрасистолы (8 эпизодов в сутки), эпизод желудочковой тахикардии (1). Для дальнейшей оценки нарушений ритма пациентке был подключен кардиомонитор.

Учитывая наличие у пациентки тяжелой гипокалиемии, артериальной гипертензии с частыми гипертоническими кризами, был заподозрен ПГА. Учитывая тяжесть состояния, гормональный статус оценивался на фоне препаратов, влияющих на РАС (периндоприл), при поступлении был начат прием АМК (спиронолактон).

Анализы крови на гормоны: АКТГ 20,64 пг/мл (N 7,2-63,3); паратгормон 57,25 пг/мл (N 15-65); ренин 0,5 мкME/мл (N 2,8-39,9); альдостерон 330 пг/мл (N 17,6-230,2).

Выполнена КТ надпочечников: образование правого надпочечника, больше в пользу аденомы, микронодулярная гиперплазия левого надпочечника.

Пациентке был назначен спиронолактон 100 мг/сутки, проводилась коррекция электролитных нарушений, продолжена гипотензивная терапия (периндоприл 8 мг/сутки, амлодипин 10 мг/сутки). На фоне лечения прошли слабость и одышка, стабилизировалось артериальное давление (120/75-140/80 мм рт. ст.), по ЭКГ и на кардиомониторе не регистрировалась экстрасистолия, улучшились показатели функции почек (калий 4,33 ммоль/л (N 3,5-5,1), креатинин 121 мкмоль/л (N 44-80), мочевина 8,4 ммоль/л (N 2,76-8,07), СКФ (CKD-EPI 51 мл/мин/1,73 м²)).

Для определения показаний к оперативному лечению пациентка была направлена на консультацию к хирургу-эндокринологу.
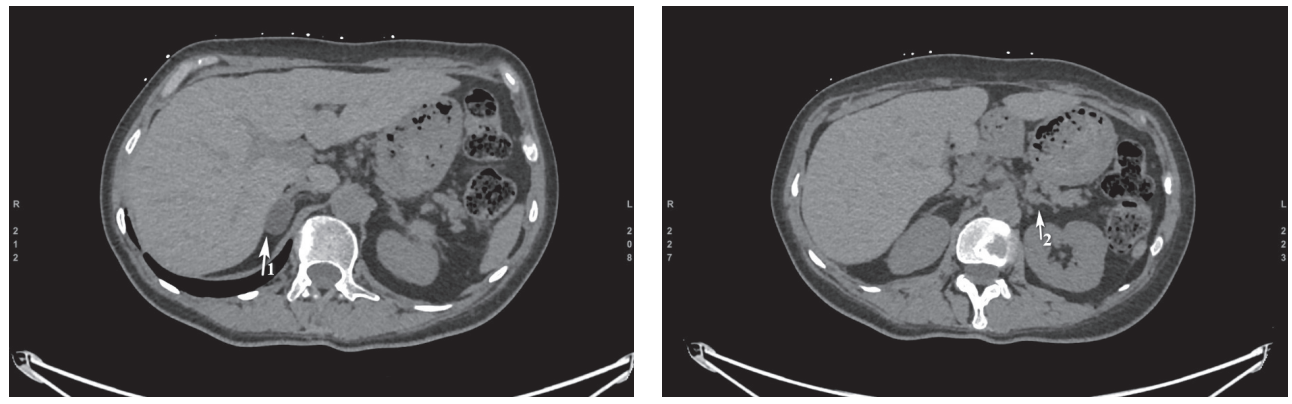

Компьютерная томография органов брюшной полости и забрюшинного пространства

Примечания: 1 - аденома правого надпочечника; 2 - микронодулярная гиперплазия левого надпочечника.

\section{CT of the abdomen and retroperitoneum}

Notes: 1 - right adrenal adenoma; 2 - micronodular hyperplasia of the left adrenal gland. 


\section{- ОБСУЖДЕНИЕ}

Данный клинический случай демонстрирует важность своевременной диагностики ПГА. Анализ медицинской документации пациентки за последние 3 года показал, что уровень калия в крови варьировал от 3,0 до 4,5 ммоль/л. Из этого следует, что гипокалиемия не является основным критерием отбора для скрининга ПГА, так как распространенность ПГА среди пациентов с умеренной и выраженной АГ без гипокалиемии оценивается в 3\% [10].

Ранняя диагностика и соответствующее патогенетическое лечение первичного гиперальдостеронизма как наиболее частой причины вторичной артериальной гипертензии позволят предотвратить неблагоприятные кардиометаболические исходы.

\section{ЗАКЛЮЧЕНИЕ}

ПГА - мультидисциплинарная проблема, в решении которой участвуют кардиологи, эндокринологи и хирурги. Отсутствие специфичных симптомов ПГА затрудняет его своевременную диагностику. Как правило, диагноз у пациентов с ПГА устанавливается к этапу формирования поражения органов-мишеней, развития тяжелой гипокалиемии. Серьезным препятствием к постановке диагноза является недостаточная настороженность терапевтов и кардиологов в отношении эндокринных АГ. В ежедневной практике врачи должны помнить о ПГА как о причине резистентной АГ. Определение альдостерон-ренинового соотношения для выявления ПГА является тестом первичной диагностики и может рекомендоваться врачами любых терапевтических специальностей.

Вклад авторов: разработка концепции и дизайна исследования, литературный обзор, описание клинического случая, редактирование текста - Казакова М.И., Галицкая С.С.

Authors' contribution: development of the concept and design of the study, collection of material, literary review, description of the clinical case, editing the text - Kazakova M., Galitskaya S.

Конфликт интересов. Авторы заявляют об отсутствии конфликта интересов.

Conflict of interest. The authors declare no conflict of interest.

\section{ЛИТЕРАТУРА/REFERENCES}

1. Shifman B.M., Platonova N.M., Molashenko N.V., Troshina E.A., Sitkin I.I., Bel'cevich D.G., Kovalevich L.D., Romanova N.Yu., Kolesnikova G.S. (2019) Pervichnyj giperal'dosteronizm: Slozhnosti diagnostiki [Primary hyperaldosteronism: difficulties in diagnostics]. Terapevticheskij arhiv, no 10.

2. Hundemer G.L., Vaidya A. (2019) Primary Aldosteronism Diagnosis and Management: A Clinical Approach. Endocrinol Metab Clin North Am., no 48 (4), pp. 681-700.

3. Monticone S., Burrello J., Tizzani D., Bertello C., Viola A., Buffolo F., Gabetti L., Mengozzi G., Williams T.A., Rabbia F., Veglio F., Mulatero P. (2017) Prevalence and Clinical Manifestations of Primary Aldosteronism Encountered in Primary Care Practice. J Am Coll Cardiol., no 69 (14), pp. 181120. doi: 10.1016/j.jacc.2017.01.052

4. Mosso L., Carvajal C., Gonzalez A., Barraza A., Avila F., Montero J., Huete A., Gederlini A., Fardella C.E. (2003) Primary aldosteronism and hypertensive disease. Hypertension, no 42 (2), pp. 161-5.

5. Rossi G.P., Bernini G., Caliumi C. (2006) A prospective study of the prevalence of primary aldosteronism in 1,125 hypertensive patients. J Am Coll Cardiol., no 48, pp. 2293-2300. 
6. Funder J.W., Carey R.M., Mantero F., Murad M.H., Reincke M., Shibata H., Stowasser M., Young W.F. Jr. (2016) The Management of Primary Aldosteronism: Case Detection, Diagnosis, and Treatment: An Endocrine Society Clinical Practice Guideline. J Clin Endocrinol Metab., no 101 (5), pp. 1889-916. doi: 10.1210/jc.2015-4061

7. Milliez P., Girerd X., Plouin P.F., Blacher J., Safar M.E., Mourad J.J. (2005) Evidence for an increased rate of cardiovascular events in patients with primary aldosteronism. J Am Coll Cardiol., no 45, pp. 1243-1248.

8. Stowasser M., Sharman J., Leano R. (2005) Evidence for abnormal left ventricular structure and function in normotensive individuals with familial hyperaldosteronism type I. J Clin Endocrinol Metab., no 90, pp. 5070-5076.

9. Reincke M., Beuschlein F., Williams T.A. (2020) Progress in Primary Aldosteronism 2019: New Players on the Block? Horm Metab Res., no 52 (6), pp. 345-346. doi: 10.1055/a-1156-9926

10. Yukina M.Yu., Troshina E.A., Belcevich D.G., Platonova N.M. (2016) Vtorichnaya (endokrinnaya) arterial'naya gipertenziya: lekciya dlya vrachej [Secondary (endocrine) arterial hypertension: lecture for doctors]. Al'manah klinicheskoj mediciny, no 44 (4), pp. 501-512.

11. Vaidya A., Mulatero P., Baudrand R., Adler G.K. (2018) The Expanding Spectrum of Primary Aldosteronism: Implications for Diagnosis, Pathogenesis, and Treatment. Endocr Rev., no 39 (6), pp. 1057-88. doi: 10.1210/er.2018-00139/.

Подана/Submitted: 16.07.2021

Принята/Accepted: 18.10.2021

Контакты/Contacts: kazakova_mariya@mail.ru,svetl_gal@mail.ru 